\title{
Enfermedades priónicas: historia, diversidad e importancia socioeconómica como paradigma de las Enfermedades Raras ${ }^{1}$
}

\section{Prion Diseases: History, Diversity, and Socioeconomic Importance as a Paradigm of Rare Diseases}

\author{
Jorge M. Charco ${ }^{2}$ \\ ORCID: https://orcid.org/0000-0003-3476-1855 \\ Tomás Barrio ${ }^{3}$ \\ ORCID: https://orcid.org/0000-0002-7037-6316 \\ Hasier Eraña ${ }^{4}$ \\ ORCID: https://orcid.org/0000-0001-8776-4211
}

Centro de investigación cooperativa en biociencias (CIC bioGUNE). ATLAS Molecular Pharma S.L.

Recibido: 08-11-2020

Aceptado: 24-12-2020

\section{Resumen}

Las enfermedades raras son aquellas patologías que afectan a una proporción muy reducida de la población (menos de 50 casos por cada 100000 personas). Por

${ }^{1}$ Este trabajo ha sido cofinanciado por el Fondo Europeo de Desarrollo Regional (FEDER) en el marco del Proyecto Interreg-POCTEFA RedPrion (EFA148/16).

2 (jmoreno.atlas@cicbiogune.es) Máster en Neurociencias por el Instituto de Castilla y León (2014) y Doctorado en Neurociencias por la Universidad del País Vasco (2018), actualmente, desarrolla su trabajo como investigador posdoctoral en el CIC bioGUNE y en la compañía Atlas Molecular Pharma, dedicado al desarrollo de terapias para enfermedades raras. Algunas de sus investigaciones han sido publicadas en revistas tales como PLOS Pathogens, Science Translational Medicine, Acta Neuropathologica, entre otras.

${ }^{3}$ (tbarrio@cicbiogune.es) Doctor en Medicina y Sanidad animal por la Universidad de Zaragoza (2019), actualmente, es investigador posdoctoral en el Prion Research Lab del CIC bioGUNE, tratando de descubrir los mecanismos que rigen la transmisión de priones. Ha publicado, entre otras, en las siguientes revistas: Scientific Reports, Molecular Neurobiology y Molecular Neurobiology.

${ }^{4}$ (herana.atlas@cicbiogune.es) Máster en Microbiología (2011) por la Universidad Autónoma de Barcelona, obtuvo su doctorado en Biología Molecular y Biomedicina por la Universidad del País Vasco en 2015. Tras un periodo como investigador posdoctoral en el CIC bioGUNE, actualmente trabaja en Atlas Molecular Pharma, compañía dedicada a la búsqueda de terapias para enfermedades raras. Entre sus investigaciones destacan las publicadas en: PLOS Pathogens, Science Translational Medicine, PNAS. 
esta razón, la investigación sobre sus causas y mecanismos, algo imprescindible para dar con una forma de tratarlas o prevenirlas, es insuficiente. Por ello, los pacientes denuncien la falta de cobertura del sistema sanitario y la discriminación social que supone padecer una de estas patologías. Entre las enfermedades raras, se encuentran las denominadas enfermedades priónicas o encefalopatías espongiformes transmisibles. A pesar de ser relativamente conocidas gracias a la crisis sanitaria que supuso el "mal de las vacas locas" a finales del siglo pasado, se conoce todavía relativamente poco sobre estas patologías que afectan tanto a animales como a humanos. En este monográfico se pretende dar a conocer la fascinante historia y la diversidad de las enfermedades priónicas, que sacudieron los cimientos de la biología conocida hasta los años 80 al poner en escena a un nuevo y desconcertante tipo de agente infeccioso: los priones.

Palabras-clave: priones, enfermedades raras, enfermedades priónicas.

\begin{abstract}
Rare disease are those pathologies that affect a reduced proportion of the population (less than 50 cases per 100000 people). For this reason, the research on their causes and mechanisms, which is essential to find a way to treat or prevent them, is insufficient. This causes that the patients report a lack of coverage by the health system and the social discrimination that suffering one of these pathologies entails. Among rare diseases, we find the so-called prion diseases or transmissible spongiform encephalopathies. Although they are relatively well-known due to the health crisis provoked by the "mad cow disease" at the end of the last century, there are still many uncertainties about these disorders that affect both animals and humans. This monograph aims at bringing to light the fascinating history and the diversity of prion diseases, which shook the foundations of the biology known before the 1980s by bringing out a new and puzzling type of infectious agent: prions.
\end{abstract}

Key-words: Prions, Rare Diseases, Prion Diseases.

\title{
Introducción
}

Las enfermedades raras (ER) o poco frecuentes se definen exclusivamente basándose en criterios de prevalencia, habiéndose establecido diversos límites en distintos países o regiones (50 casos por 100000 habitantes en Europa o 80 casos por 100000 habitantes en EEUU). No obstante, a pesar de la aparente simplicidad de su definición, las ER son muy numerosas, heterogéneas y geográficamente dispersas: según la base de datos Orphanet, 
existen más de 6000 ER distintas, entre las cuales hay muy pocas que sean prevenibles, tratables o curables, lo que hace que sean mayoritariamente enfermedades crónicas o causantes de muerte prematura (Nguengang Wakap et al., 2020). A pesar de esta heterogeneidad, todas las ER comparten una serie de características derivadas precisamente de su baja prevalencia en la población: una naturaleza crónica, debilitante o degenerativa y en muchos casos inevitablemente letal, un conocimiento generalmente muy escaso de sus causas y mecanismos moleculares, y una escasez de investigación relacionada debida a los bajos números de afectados. Por este mismo motivo, los afectados por una ER denuncian también las dificultades y tardanza en recibir un diagnóstico correcto, la baja o nula cobertura de productos sanitarios por la Sanidad Pública, la discriminación social que padecen o el descuido por parte de las autoridades de apoyo a sus necesidades especiales de dependencia o de integración laboral (FEDER, n.d.).

Sin embargo, dado que en conjunto pueden llegar a afectar a una parte importante de la población (se estima que en Europa afectan a entre un $6 \mathrm{y}$ un $8 \%$ de la población) (European Commission, n.d.), en los últimos años las enfermedades raras han atraído la atención de las instituciones gubernamentales, que han empezado a otorgarles un lugar especial al diseñar políticas de salud pública, considerándolas prioritarias y desarrollando legislación específica para facilitar su investigación y el desarrollo de potenciales terapias.

En este contexto, es especialmente importante dar visibilidad y mejorar la percepción social de las ER tanto en su conjunto como individualmente, con el fin de estimular mejoras en las políticas públicas y fomentar su investigación, que es, en última instancia, la única vía para ofrecer esperanza a los millones de afectados por estas patologías. A través de este monográfico, se pretende avanzar en este objetivo aportando información acerca de las encefalopatías espongiformes transmisibles (EET) o enfermedades priónicas, un pequeño grupo de enfermedades relacionadas que son especialmente raras no sólo por el reducido número de afectados, sino también por su particular etiología. Este grupo de enfermedades, más conocido gracias al tremendo impacto socioeconómico que tuvo la crisis del mal de las vacas locas en Europa en los años 90 (Verbeke, 2001), ilustra perfectamente los problemas a los que se enfrentan aquellos afectados por enfermedades raras, como el limitado conocimiento, la reducida investigación, las dificultades para un correcto diagnóstico o la discriminación social, acentuada en este caso por el carácter transmisible o infeccioso de algunas EET. Por ello, a continuación, vamos a realizar un repaso a la historia del descubrimiento de estas ER y sus principales características con la intención de incrementar su visibilidad y acercar la problemática de las ER a la sociedad. 


\section{La historia del descubrimiento de los priones}

Una de las peculiaridades de las enfermedades priónicas es la controversia que ha rodeado durante décadas la naturaleza de su agente causal. ¿Qué es responsable de la muerte neuronal? ¿Por qué adquiere ese aspecto de esponja el cerebro, como se evidencia bajo la luz del microscopio? Para contestar a estas preguntas, nos tenemos que remontar a la Inglaterra del siglo XVIII, donde se documenta por primera vez la existencia de una enfermedad priónica. A través de una carta al Parlamento de Inglaterra, los ganaderos de la región de Lincolnshire exigían un control más exhaustivo del ganado ovino procedente de España, puesto que muchos de los animales estaban afectados de tembladera (o scrapie, que es como se conoce a esta enfermedad en inglés). Ya en esa época se conocía que la enfermedad podía "saltar" de unas ovejas a otras, ya que, tras la introducción de un animal infectado en un rebaño, era común que otros animales mostraran los mismos síntomas (temblores, picores y pérdida de lana en diferentes zonas del cuerpo). A lo largo del siglo XIX, se formularían diferentes teorías acerca de la naturaleza del agente que afectaba a estos animales. Por ejemplo, se relacionó con las tormentas eléctricas o con la sobreactividad sexual, aunque sin ninguna base científica. Ya en el siglo XX, se demostró de forma inequívoca que la enfermedad estaba causada por un agente infeccioso (Brown, 2009), como ya se sospechaba en siglos previos. Esto, sumado a los largos periodos de incubación y a la evidencia de que se trataba de un agente filtrable, señalaron como principal sospechoso a un virus lento que afectaba al sistema nervioso central.

A la vez que se investigaban las causas de la tembladera, dos neurólogos, Hans Gerhard Creutzfeldt y Adolf Jakob, describieron en 1920 una extraña enfermedad neurológica, que más tarde el neurólogo alemán Spielmeyer bautizaría como enfermedad de Creutzfeldt-Jakob (Creutzfeldt, 1920; Jakob, 1921). Entre los síntomas estaban graves problemas motores y un evidente retraso cognitivo. En todos los casos, los pacientes terminaban falleciendo. Varias décadas después esta enfermedad se relacionaría con el scrapie, siendo por tanto la primera encefalopatía espongiforme descrita en humanos. Sin embargo, no sería la enfermedad de Creutzfeldt-Jakob la que nos daría la pista para aunar todas estas patologías bajo el paraguas de las enfermedades priónicas. Las claves que desentrañarían el misterio acerca del agente causal de estas enfermedades se empezarían a gestar con el viaje del doctor Vincent Zigas a Papúa-Nueva Guinea en la década de 1950. El doctor Zigas, que había sido nombrado oficial médico de la zona, fue el primer occidental en describir una enfermedad muy peculiar entre los miembros de la etnia fore. Para ellos, que apenas hacía 10 años que habían entrado en contacto con los occidentales, esta enfermedad se trataba de un embrujo o mal de ojo formulado por un 
chamán. Los miembros embrujados de la tribu sufrían de terribles temblores y graves dificultades para caminar. Esta enfermedad se conocía como Kuru, que en el idioma de los fore significa temblar de miedo o frío. Finalmente, el embrujado terminaba postrado en la cama y moría irremediablemente. De inmediato, el doctor Zigas envió varias cartas a sus colegas y a diferentes foros médicos describiendo lo que para él era una extraña encefalitis que afectaba exclusivamente a los miembros de esta etnia. De esta forma, varios antropólogos y médicos viajaron a la isla para investigar esta misteriosa encefalitis (Liberski et al., 2019). Entre los médicos que arribaron a Papúa-Nueva Guinea para estudiar el Kuru estaba el doctor Carleton Gajdusek. Al poco tiempo de llegar a la isla y entrar en contacto con los fore, el doctor Gajdusek observó que los niños y las mujeres eran los que sufrían la enfermedad con mayor frecuencia, hecho que había pasado desapercibido hasta ese momento. Obviamente, el doctor Gajdusek no creía que estuviera relacionada con ningún embrujo y empezó a investigar el día a día de los fore. ¿Qué podía explicar una encefalitis que afectaba sobre todo a los niños y las mujeres si estos convivían con los hombres adultos y se alimentaban igual que ellos? La respuesta se encontraba al parecer en una oscura ceremonia que los fore realizaban como rito mortuorio cuando un hombre de la tribu fallecía. Durante estos ritos, varios miembros de la tribu cocinaban y comían el cuerpo del fallecido, de forma que su espíritu fuera liberado. Pero fue una parte muy concreta de este ritual canibalístico la que llamo especialmente la atención del doctor Gajdusek: en muchas ocasiones, el encéfalo de los hombres fallecidos era consumido exclusivamente por la mujer y los hijos de este. Así, encontró una relación entre el canibalismo ritual y la mayor prevalencia del Kuru en niños y mujeres, y por lo tanto pudo concluir que el hecho de consumir el encéfalo de un fallecido estaba estrechamente relacionado con el desarrollo de la enfermedad. Esto reforzaba la hipótesis de que un virus lento que afectaba al sistema nervioso era el responsable del Kuru, ya que el consumo de partes del individuo donde estuviera presenta este virus podría contagiar al consumidor (Gajdusek, 1967). Durante los siguientes años, el doctor Gajdusek dedicó gran parte de sus esfuerzos a aislar e identificar este virus en los encéfalos de varios miembros de la tribu fore "embrujados" de Kuru. Sin embargo, nunca sería capaz de encontrar el escurridizo virus responsable de esa misteriosa enfermedad. A pesar de ello, el doctor Gajdusek recibiría el premio Nobel de Fisiología y Medicina en 1976 por sus descubrimientos.

Pasados varios años, una situación totalmente fortuita llevaría a la relación del scrapie con el Kuru y al inicio del estudio de las encefalopatías espongiformes, incluyendo ya la enfermedad de Creutzfeldt-Jakob, como un conjunto de síndromes con el agente causal como nexo común. El responsable fue el doctor William Hadlow, un joven patólogo veterinario que había comenzado a estudiar el scrapie en los Rocky Mountain Laboratories de los Institutos Nacionales de la 
Salud de EEUU. Hadlow, que se encontraba en Inglaterra con motivo de una serie de ponencias, acudió a una conferencia impartida por el doctor Gajdusek acerca del misterioso mal de los fore. Allí, pudo observar las lesiones de los encéfalos de esos pacientes. En ellas se apreciaban grandes espacios vacíos, llamados vacuolas, tanto dentro como fuera de las neuronas. Estas vacuolas daban aspecto de esponja al tejido, lo que inmediatamente llamó la atención del doctor Hadlow, ya que estas lesiones eran extremadamente parecidas a las que él mismo observaba cada día en las ovejas afectadas por la tembladera. Hadlow comenzó a seguir más de cerca el trabajo de Gajdusek y poco después relacionó ambas enfermedades indicando que podrían estar provocadas por el mismo agente (Hadlow, 2008), es decir, que se trataba de la misma enfermedad y que esta podía afectar tanto a las ovejas como a los humanos. En 1966, Gajdusek y Gibbs demostraban que el Kuru era infeccioso en chimpancés, evidenciando de forma definitiva que se trataba de una enfermedad infecciosa estrechamente relacionada con el scrapie (Gajdusek et al., 1967). Tan sólo dos años después de la publicación de los chimpancés infectados de Kuru, el doctor Klatzo relacionaría la enfermedad de Creutzfeldt-Jakob con el scrapie y con el Kuru (Klatzo et al., 1959). Esta relación se cimentó, en gran medida, en la asombrosa similitud de los síntomas y las lesiones espongiformes del encéfalo de pacientes de esta enfermedad con los de Kuru o las ovejas afectadas de scrapie. La repetición del experimento llevado a cabo con el Kuru en chimpancés con material infectado de pacientes de enfermedad de Creutzfeldt-Jakob (Gibbs et al., 1968) terminó por demostrar que esta también era una encefalopatía transmisible y, por tanto, estaba estrechamente relacionada con el Kuru y el scrapie.

Durante la segunda mitad del siglo $\mathrm{XX}$ se describieron varias encefalopatías espongiformes que afectaban a humanos y a otros mamíferos. Todos los casos presentaban cambios espongiformes en el sistema nervioso central, se trataba de enfermedades transmisibles y, en la mayoría de los casos, se observaban problemas motores y cognitivos. En humanos se describió el insomnio familiar fatal, la enfermedad de Gerstmann-Straüssler-Scheinker y la prionopatía con sensibilidad variable a proteasas. En otros mamíferos destacan, por su importancia socioeconómica, la encefalopatía espongiforme bovina, la enfermedad debilitante crónica de los cérvidos y el scrapie, ya mencionado. En este monográfico dedicaremos un apartado a todas ellas, indicando sus características más importantes.

\section{¿Virus lento o...?}

Ya conocemos el principio de la historia de las enfermedades priónicas. Sabemos qué síndromes se engloban dentro de este grupo de enfermedades y 
cuáles son sus características comunes. Sin embargo, todavía no sabemos cuál es su agente causal. Como hemos indicado anteriormente, al tratarse de una enfermedad infecciosa con un largo periodo de incubación y sabiendo que el agente es filtrable (Gajdusek et al., 1966), durante décadas la principal teoría fue la del virus lento. Sin embargo, conforme avanzaban los conocimientos en biología y se mejoraban las técnicas analíticas, se fue constatando que la teoría del virus lento podía no ser acertada. Por ejemplo, estudios realizados por la doctora Tikvah Alper demostraron que el supuesto virus era resistente a la radiación ultravioleta (Alper, 1972). Esto era difícil de creer, puesto que esta radiación debería destruir totalmente el material genético contenido en cualquier virus. Además, trató de estudiar su tamaño mediante filtraciones sucesivas, y llegó a la conclusión de que era más pequeño que cualquier virus conocido. Por lo tanto, o el virus era extremadamente pequeño y resistente a la radiación, o no se trataba de un virus. La destrucción del material genético del virus debería terminar por desactivarlo completamente, por lo que muchos investigadores fijaron éste como su objetivo prioritario. Sin embargo, todos los intentos fueron infructuosos y se demostró que el virus era totalmente inmune a la inactivación por nucleasas (enzimas que destruyen el material genético) o cualquier otro método conocido de inactivación de ácidos nucleicos (Latarjet et al., 1970). Todos estos datos abrieron la puerta a una nueva teoría propuesta por el doctor Stanley Prusiner, juntando las pequeñas pistas obtenidas por varios investigadores y por él mismo a lo largo de décadas. Sin embargo, y como veremos más adelante, la confirmación de esta teoría sacudiría los cimientos de la biología.

\section{Un pequeño inciso para aclarar conceptos de bioquímica}

Las proteínas son unas moléculas de gran complejidad formadas por pequeños bloques unidos de forma secuencial, formando una cadena continua. Tenemos 20 de estos bloques distintos llamados aminoácidos, y dependiendo de cómo se ordenen estos bloques tendremos una cadena diferente. Al tener tantos bloques diferentes disponibles, y teniendo en cuenta que puede haber miles de estos bloques en una sola proteína, las combinaciones que podemos generar son prácticamente infinitas. La única regla es que cada bloque sólo está unido a los dos que tiene al lado, como los eslabones de una cadena. Sin embargo, existen interacciones débiles que hacen que unos eslabones tiendan a acercase a otros, aunque se encuentren lejanos en la cadena. Este fenómeno hace que la cadena se pliegue sobre sí misma de una forma concreta dependiendo de los bloques que tengamos en la cadena. Es decir, si tengo dos proteínas formadas por los mismos bloques colocados en el mismo orden, 
ambas se plegarán de la misma forma. Esta característica de las proteínas es muy importante, puesto que dependiendo de cómo se hayan plegado, tendrán una función u otra (transportar oxígeno, transformar glucosa en energía, formar parte de los huesos, el músculo, etc). Pero ¿dónde está la información que dicta el orden correcto de los bloques para formar una proteína concreta? En todos los organismos vivos existen miles de proteínas diferentes y un pequeño fallo en cualquiera de ellas puede resultar fatal, puesto que muchas de ellas realizan funciones vitales. Por ello, la información para formar todas estas proteínas debe transmitirse de una forma fiel e infalible. El portador de esta información es el material genético, formado por los ácidos nucleicos, que contienen las instrucciones para dar lugar a cada uno de los aminoácidos y el orden en el que se deben colocar para generar los miles de proteínas existentes en todos los seres vivos del planeta.

Así, ya tenemos claros varios conceptos de biología que son muy importantes para entender la enorme controversia que provocó la teoría de Prusiner: i) las proteínas están formadas por aminoácidos. ii) El orden de estos aminoácidos determina cómo se pliega la proteína, lo que a su vez determina su función. iii) Dos proteínas con los mismos aminoácidos colocados en el mismo orden tienen el mismo plegamiento y, por tanto, la misma función. iv) Los ácidos nucleicos codifican la información necesaria para que los aminoácidos se coloquen de la forma correcta en una proteína. v) Toda la información se encuentra contenida en los ácidos nucleicos. Por lo tanto, debemos recordar que proteína es igual a función y ácidos nucleicos es igual a información. Estos conceptos forman parte de lo que se conoce como dogma central de la biología, que a su vez forma parte de una serie de normas que todos los seres vivos del planeta siguen sin excepción.

\section{Entonces... ¿Qué es un prion?}

Según la teoría formulada por el doctor Prusiner en 1982, un prion (del inglés Proteinaceous Infectious Particle) es una proteína plegada de una forma extraña, no nativa, que es capaz de transformar a otras proteínas, haciéndolas adoptar su misma forma (Prusiner, 1982). Es decir, se trata de una proteína que, aunque tiene los mismos bloques en el mismo orden, puede plegarse de, al menos, dos formas diferentes y, por tanto, tener funciones diferentes. Ya de entrada, Prusiner estaba violando uno de los dogmas de la biología. Según esta teoría, la $\operatorname{PrP}^{\mathrm{Sc}}$ (de scrapie) es una forma aberrante de la proteína priónica $\mathrm{PrP}^{\mathrm{C}}$ (de celular). La $\mathrm{PrP}^{\mathrm{C}}$ es una proteína de función desconocida que se encuentra de forma ubicua en todos los mamíferos. Sin embargo, bien sea por la presencia de diferentes mutaciones, bien por una entrada de $\mathrm{PrP}^{\mathrm{Sc}}$ en el 
organismo procedente del exterior, o bien de forma espontánea (es decir, por un proceso desconocido), la $\operatorname{PrP}^{\mathrm{C}}$ sana, normal, de nuestro cerebro se transforma en $\mathrm{PrP}^{\mathrm{Sc}}$. Ahora esta $\mathrm{PrP}^{\mathrm{Sc}}$ es capaz de transformar otras $\mathrm{PrP}^{\mathrm{C}}$ en $\mathrm{PrP}^{\mathrm{Sc}}$ por un proceso también desconocido. Así, poco a poco va aumentando la cantidad de $\operatorname{PrP}^{\mathrm{Sc}}$ en el sistema nervioso central (de ahí que la enfermedad sea tan lenta). Otra característica de esta $\mathrm{PrP}^{\mathrm{Sc}}$ es que tiene una alta neurotoxicidad. Es decir, daña las neuronas, que al morir dejan unos huecos (llamados vacuolas) en el tejido nervioso, lo que da al cerebro ese aspecto esponjoso cuando se mira al microscopio, siendo ésta una de las características definitorias de las encefalopatías espongiformes.

La teoría de Prusiner, que parece tan simple, supuso la ruptura de muchas de las reglas que creíamos definitorias de la vida en el planeta tal y como la conocemos. Por ejemplo, esta teoría implica que la $\mathrm{PrP}^{\mathrm{Sc}}$ es capaz de transmitir una información, en este caso un cambio en el plegamiento y su consiguiente cambio de función, a otra proteína. Por si esto fuera poco, la teoría priónica erosionaba aún más la regla que indicaba que dos proteínas con los mismos aminoácidos en el mismo orden debían dar lugar al mismo plegamiento proteico y, por tanto, tener las mismas funciones. Ya se conocía desde mediados de los años 60 la existencia de cepas del que por aquel entonces se consideraba un virus lento (Pattison \& Millson, 1961). Con cepas nos referimos a virus muy similares, pero con una serie de mutaciones (cambios en su material genético) que le aportan ciertas características distintivas (mayor o menor virulencia, mayor o menor resistencia a antivirales, etc). Es decir, un cambio en su material genético se plasma en un cambio en sus proteínas, adquiriendo nuevas características. Sin embargo, ¿cómo es esto posible si, según la teoría priónica, no tenemos un virus, sino una proteína que simplemente ha cambiado su plegamiento sin la presencia de material genético? Lo que propone la teoría es que estos cambios se deben a diferentes plegamientos de la $\mathrm{PrP}^{\mathrm{Sc}}$. Estos plegamientos se mantienen cuando infectamos a nuevos individuos. Es decir, que estamos transmitiendo información a través del plegamiento de la proteína. Así que, por si no fuera poco el cambio de $\operatorname{PrP}^{\mathrm{C}}$ a $\mathrm{PrP}^{\mathrm{Sc}}$, resulta que la $\mathrm{PrP}^{\mathrm{Sc}}$ es capaz de transmitir información detallada de su estructura a la $\operatorname{PrP}^{\mathrm{C}}$, generando decenas de cepas diferentes. Esto sí que era un desafío directo a las normas por aquel entonces conocidas de la biología, ya que se estaba hablando de transmisión de información sin material genético.

Por supuesto, Prusiner fue inicialmente denostado por la comunidad científica al atreverse a desafiar el dogma. De esta forma, comenzó una batalla científica con el objetivo de demostrar si la existencia de una proteína capaz de portar información era o no era posible. A lo largo de las décadas de los 80, 90 y principios del 2000, diferentes grupos de investigación fueron encontrando numerosas pruebas de que Prusiner estaba en lo cierto. Por ejemplo, se pudo 
demostrar que animales sin $\operatorname{PrP}^{\mathrm{C}}$ eran totalmente inmunes a la enfermedad (Bueler et al., 1993). También se demostró que se podía generar $\operatorname{PrP}^{\mathrm{Sc}}$ en un tubo de ensayo en el que sólo había $\operatorname{PrP}^{\mathrm{C}}$ y algunos componentes celulares o artificiales (Saborio et al., 2001). Sin embargo, la confirmación total de la teoría llegaría en 2010, con la generación de un prion totalmente artificial utilizando exclusivamente $\operatorname{PrP}^{\mathrm{C}}$ generada en una bacteria (Wang et al., 2010). En este experimento se consiguió generar un prion capaz de infectar animales de la misma forma que ocurriría si usáramos $\operatorname{PrP}^{\mathrm{Sc}}$ procedente de otro animal. Por tanto, si podía simularse lo que ocurre en la naturaleza sin la inclusión de ningún material genético, quedaba totalmente confirmada la existencia de los priones como proteínas con plegamientos diferentes capaces de modificar a otras proteínas y transmitirles una información sin la existencia de ningún material genético. Hoy en día la teoría de Prusiner se acepta sin reservas y, de hecho, se ha visto expandida por diferentes pruebas que sugieren que varias enfermedades neurodegenerativas podrían tener su origen en mecanismos similares de transmisión de información entre proteínas. Son las que se conocen como enfermedades prion-like, y entre ellas tenemos la enfermedad de Alzheimer, la enfermedad de Parkinson, la esclerosis lateral amiotrófica o la amiloidosis sistémica (Eraña et al., 2017). También se ha encontrado que este tipo de transmisión de información ocurre en bacterias y levaduras, aunque con una finalidad radicalmente diferente (Wickner et al., 2015; Yuan \& Hochschild, 2017). Finalmente, y tras luchar contra viento y marea para demostrar que la Proteinaceous Infectious Particle (prion) existía, el doctor Stanley Prusiner fue galardonado con el premio Nobel de Medicina y Fisiología en 1997.

\section{Enfermedades priónicas en humanos}

Los seres humanos pueden sufrir distintas formas de enfermedad priónica, caracterizadas por cuadros clínicos muy diversos y por la afectación de áreas concretas del sistema nervioso. Así, la enfermedad de Creutzfeldt-Jakob (CJD) se presenta clínicamente como una demencia de progresión rápida (usualmente de unas pocas semanas o meses) acompañada de signos neurológicos (mioclonías, ataxia cerebelar, alteraciones de la visión) (Johnson \& Gibbs, 1998). Por su parte, el insomnio fatal, una enfermedad caracterizada por una afectación fundamentalmente talámica, cursa con insomnio y alteraciones del sueño (Kretzschmar et al., 1995). Por último, el síndrome de GerstmannSträussler-Scheinker (GSS), además de presentar un perfil neuropatológico muy característico, se manifiesta clínicamente con síntomas de disfunción cerebelar (ataxia, nistagmo, disartria) (Takada et al., 2017). 
Esta clasificación, basada en criterios clínicos y neuropatológicos, se emplea en el ámbito de las ciencias de la salud para dar un pronóstico del paciente y orientar los posibles tratamientos paliativos. Sin embargo, desde el punto de vista de la patogenia, es más útil clasificar las enfermedades priónicas atendiendo a su origen, es decir, al evento que provoca el malplegamiento inicial de la $\operatorname{PrP}^{\mathrm{C}}$. Así, las EET pueden dividirse en: 1) esporádicas, 2) genéticas y 3) adquiridas.

\section{1) Enfermedades priónicas esporádicas en humanos}

Las EET de tipo esporádico suponen la gran mayoría (80-85 \%) de los casos de enfermedad priónica en seres humanos (Hill et al., 2003). En estos casos esporádicos, la causa del malplegamiento inicial de la $\operatorname{PrP}^{\mathrm{C}}$ no se ha discernido todavía. Se sospecha que puede ser un evento espontáneo y extremadamente raro de cambio de conformación (Cohen \& Prusiner, 1998). Sin embargo, existen autores que proponen hipótesis diferentes: por ejemplo, algunos sugieren que una mutación somática (es decir, un cambio genético no heredado, que ocurre en una única célula del organismo de forma aislada) podría desencadenar el malplegamiento de las $\operatorname{PrP}^{\mathrm{C}}$ mutadas que, una vez iniciado, podría propagarse a las $\mathrm{PrP}^{\mathrm{C}}$ no mutadas (Prusiner, 1989). Por último, a pesar de la falta de evidencias epidemiológicas, algunos investigadores señalan la posibilidad de que estos casos aparentemente esporádicos sean debidos a una exposición a bajo nivel a priones de origen externo (Linsell et al., 2004).

El ejemplo más importante de las EET de tipo esporádico es la forma esporádica de la enfermedad de Creutzfeldt-Jakob (sCJD). Su prevalencia en la población está en torno a 1-2 casos por cada millón de personas al año (Hill et al., 2003), por lo que, de acuerdo con los criterios expuestos anteriormente (European Commission, n.d.), se considera una enfermedad rara.

Existen otras dos enfermedades priónicas esporádicas que se dan en los seres humanos, aunque su frecuencia de aparición es mucho menor que la de la sCJD. La primera se denomina insomnio fatal esporádico (sFI). Se trata de una patología clínicamente indistinguible del insomnio familiar fatal (FFI), una EET de origen genético de la que hablaremos después. Sin embargo, los casos de sFI aparecen de forma esporádica, no vinculados con la mutación causal del FFI (Gambetti et al., 2003).

La otra EET esporádica que encontramos en humanos es la prionopatía de sensibilidad variable a las proteasas (VPSPr), que fue descrita recientemente (Gambetti et al., 2008). A pesar de que se desconocen los detalles sobre su origen tanto como se desconocen los de la $\mathrm{SCJD}$, las características neuropatológicas y bioquímicas de esta enfermedad ha llevado a algunos autores a considerarla como la forma esporádica del GSS (Notari et al., 2014), del que también hablaremos en los siguientes párrafos. 


\section{2) Enfermedades priónicas genéticas en humanos}

Entre el 10 y el $15 \%$ de los casos de enfermedad priónica en humanos son casos genéticos o familiares (Kovacs et al., 2005). En las EET de tipo genético, el gen que codifica la $\mathrm{PrP}^{\mathrm{C}}$ contiene una mutación, es decir, un error en su secuencia que provoca el cambio de un aminoácido de la proteína por otro distinto. Esto hace que la $\operatorname{PrP}^{\mathrm{C}}$ sea más propensa a malplegarse a $\operatorname{PrP}^{\mathrm{Sc}} \mathrm{y}$, por lo tanto, a provocar la enfermedad. Dependiendo de en qué aminoácido se dé la mutación, las características de la enfermedad resultante pueden variar ampliamente.

En los seres humanos existen más de 50 mutaciones en el gen de la $\operatorname{PrP}^{\mathrm{C}}$ que han sido relacionadas con el desarrollo de una EET. Sin embargo, el 85 $\%$ de todos los casos de EET genéticas descritas se deben a tan solo cinco mutaciones: E200K, V210I y V180I, asociadas a las formas familiares de CJD (fCJD); D178N, la mutación responsable del insomnio familiar fatal, y P102L, la mutación más comúnmente encontrada en los casos de síndrome de Gerstmann-Sträussler-Scheinker (Minikel et al., 2016; Takada et al., 2017).

Mientras que la fCJD es clínicamente similar a los casos esporádicos de CJD, el FFI cursa con alteraciones del sueño, consecuencia de una fuerte afectación de la región talámica. Como hemos dicho, esta enfermedad está causada por la mutación D178N, pero sólo cuando ésta aparece ligada a una metionina en la posición 129; en caso contrario, lo que se desarrolla es un tipo de fCJD (Kretzschmar et al., 1995).

Por su parte, el síndrome de Gerstmann-Sträussler-Scheinker (GSS) se distingue a nivel clínico por una sintomatología que indica afectación cerebelar. En realidad, constituye un conjunto heterogéneo de patologías causadas por distintas mutaciones localizadas a lo largo de la proteína (Tesar et al., 2019). Se han descrito 16 mutaciones puntuales asociadas al GSS, siendo la más común la P102L (Takada et al., 2017).

\section{3) Enfermedades priónicas adquiridas en humanos}

La última clase de EET son las de tipo adquirido. Éstas están provocadas por priones de origen externo, que penetran en el organismo por distintas vías, frecuentemente la vía oral. Debido a las estrictas medidas de control que están actualmente vigentes, este tipo de casos son muy poco frecuentes en los humanos, suponiendo menos del $1 \%$ de los casos totales. Sin embargo, las EET adquiridas continúan siendo las más importantes en animales, como veremos en secciones posteriores. Existen tres EET adquiridas que se dan en humanos: la enfermedad de Creutzfeldt-Jakob iatrogénica (iCJD), la variante de la enfermedad de Creutzfeldt-Jakob (vCJD), y el Kuru, del que ya se ha hablado y que a día de hoy se considera erradicada. 
En la iCJD, como se propio nombre indica, los priones penetran el organismo por causas iatrogénicas. Los procedimientos médicos responsables de estos casos fueron los trasplantes de duramadre o de córnea procedentes de pacientes de CJD (Duffy et al., 1974; Thadani et al., 1988), el tratamiento con hormona de crecimiento extraída de cadáveres con priones (Bonda et al., 2016) y el uso de material quirúrgico contaminado con estos agentes (Bonda et al., 2016; Brown et al., 2012). No obstante, estos contagios ocurrieron hace décadas, cuando todavía no se conocía la capacidad de transmisión entre individuos de estas patologías. Debido al desuso de hormona de crecimiento de origen cadavérico y gracias al control de los trasplantes de duramadre y córnea y a la aplicación de medidas de desinfección del material quirúrgico, puede considerarse que el riesgo de contraer iCJD en nuestros días es mínimo (Brown et al., 2012).

A finales del siglo pasado, la aparición de la variante de la CJD (vCJD) en seres humanos y su vinculación con la encefalopatía espongiforme bovina (Hill et al., 1997) marcaron un antes y un después en la investigación en priones. La aparición en el Reino Unido, a principios de la década de 1990, de los primeros casos de una nueva enfermedad neurológica, similar a la CJD pero que seguía un patrón de distribución en la población sugestivo de una causa infecciosa (Collinge et al., 1996), disparó todas las alarmas. Tras años de investigación, se concluyó que estos casos en humanos estaban causados por la ingestión de productos cárnicos procedentes de vacas aquejadas de encefalopatía espongiforme bovina (EEB o BSE, por sus siglas en inglés). Esta enfermedad, de la que hablaremos más adelante, saltó a los medios de comunicación con el nombre de "mal de las vacas locas" y fue responsable de una crisis alimentaria sin precedentes en la Unión Europea, con enormes repercusiones a nivel sanitario, económico y político (Verbeke, 2001).

Los casos de vCJD en pacientes humanos se caracterizan por una presentación clínica que se inicia con síntomas psiquiátricos, por aparecer en individuos mucho más jóvenes y por una progresión de la patología más lenta, en comparación con los casos de sCJD y los casos genéticos (Glatzel et al., 2005) Gracias al estricto control de los productos de origen bovino que se impuso en los mataderos y las industrias cárnicas, por un lado, y a las medidas de control veterinario orientadas a detectar y erradicar la enfermedad en el ganado bovino, por otro, en la actualidad el riesgo de contraer esta enfermedad por consumo de carne contaminada es prácticamente inexistente. 


\section{Enfermedades priónicas en animales}

Las enfermedades priónicas no suponen solamente un reto en medicina humana: también son importantes en el ámbito de la salud animal. Los veterinarios están familiarizados con estas patologías desde el siglo XVIII, de cuando datan las primeras descripciones en Reino Unido de la enfermedad de scrapie en ovejas de raza Merina (Liberski, 2012). Esta enfermedad también es conocida en español como "tembladera" y "prurigo lumbar", en alusión a los signos clínicos más frecuentes que presentan los ovinos: temblores, alteraciones de la movilidad y prurito. Además de ser la EET más antigua conocida, es también la más ampliamente estudiada: fue con el scrapie que se iniciaron las investigaciones sobre la capacidad de transmisión de estas patologías, primero de oveja a oveja (Cuille \& Chelle, 1936), y más tarde a ratones (Chandler, 1961), ratas y hámsteres (Zlotnik \& Rennie, 1965). También fue esta enfermedad la que llevó por primera vez al aislamiento de la proteína prion patológica, al descubrimiento de su homóloga celular y, con ello, al establecimiento de la hipótesis "solo proteína" por Stanley Prusiner (Prusiner, 1982). Por todo ello, el scrapie se considera, en muchos aspectos, el prototipo de las EET.

En el ganado bovino, por su parte, la emergencia de la encefalopatía espongiforme bovina (EEB o BSE) en la Europa de finales de los 80 y su acertada vinculación con lo que se denominó "nueva variante de la enfermedad de Creutzfeldt-Jakob (vCJD) (Hill et al., 1997) conllevó (como se recoge en párrafos anteriores) una crisis sanitaria y económica sin precedentes (Verbeke, 2001). Clínicamente, la enfermedad se caracteriza sobre todo por cambios en el comportamiento (nerviosismo y agresividad) (Konold et al., 2004), lo que le valió la denominación popular de "mal de las vacas locas".

Existen evidencias de que la BSE pudo derivar originalmente de priones de origen ovino. En un estudio muy reciente (Huor et al., 2019) se vio que los encéfalos de ovejas que desarrollaron de forma espontánea scrapie atípico contenían pequeñas cantidades de priones de BSE, que pudieron aislarse y amplificarse empleando técnicas y modelos animales específicos. Estos resultados alertan sobre la posibilidad de que la historia pueda volver a repetirse si se relajan las medidas impuestas por la Unión Europea para el control de las EET animales (como, por ejemplo, la prohibición de usar proteína extraída de subproductos animales para la alimentación de los rumiantes). Por ello, se recomienda cautela y el mantenimiento de las políticas de vigilancia y control de esta y otras EET animales, en tanto los misterios en torno al origen y los mecanismos de transmisión y divergencia de estas enfermedades no se resuelvan por completo.

Por su parte, al igual que los rumiantes, los cérvidos también están afectados por las enfermedades priónicas. En 1967 en el estado de Colorado (Estados 
Unidos) se identificó un ciervo mula (Odocoileus hemionus) doméstico aquejado por una enfermedad neurodegenerativa nunca vista. El animal presentaba una pérdida de peso progresiva y un comportamiento inusual, que lo llevaba a aislarse del resto del rebaño (Mathiason, 2017). Poco después aparecieron más animales afectados, tanto domésticos como de vida libre y tanto de la misma como de distintas especies de cérvidos, y la enfermedad poco a poco se extendió por diversos estados de Estados Unidos y por Canadá, alcanzando prevalencias muy altas (Williams, 2005). Esta nueva enfermedad se bautizó como enfermedad debilitante crónica (CWD por sus siglas en inglés) y, tras el estudio de los casos, se incluyó dentro del grupo de las EET. La CWD llegó también hasta Corea del Sur y, en el año 2016, se describió por primera vez en Europa, inicialmente en Noruega (Benestad et al., 2016), y más tarde en países vecinos. El hecho de que sea la única enfermedad priónica que afecta tanto a animales domésticos como silvestres, su gran capacidad de diseminación, la persistencia del agente en el ambiente y el desconocimiento que existe sobre su capacidad de transmisión a humanos (Houston \& Andreoletti, 2019) hacen que el seguimiento y control de la CWD sea un tema de la mayor importancia en la actualidad (Duque Velasquez et al., 2015)

\section{Repercusión y dificultades sociosanitarias en las enfermedades prióni- cas y otras enfermedades raras}

Las características tan particulares de las enfermedades priónicas hacen de las mismas un buen ejemplo de, al menos, una parte de las dificultades asociadas al padecimiento de una ER a nivel social y sanitario.

Debido a su carácter transmisible, los casos de EET son de declaración obligatoria en muchos países y se realiza un seguimiento epidemiológico de los mismos tanto en animales como en seres humanos. Es por ello que, a diferencia de lo que ocurre con la mayoría de ER, se dispone de numerosos datos epidemiológicos que facilitan la realización de estudios retrospectivos. Aunque esto suponga una ventaja frente a otras ER para las que no existen registros públicos, también añade una dimensión de discriminación social más allá de lo que padecen otros pacientes afectados por patologías poco comunes. Según datos de la Federación Española de Enfermedades Raras (FEDER), hasta un $75 \%$ de sus asociados ha sentido discriminación debido a su enfermedad en ámbitos diversos, destacando comportamientos discriminatorios incluso en el ámbito sanitaria (32\%). Esto es debido a que estas ER pueden confundirse con enfermedades más comunes y a que, además, tienen un componente infeccioso. En el caso particular que nos ocupa, un análisis del impacto social llevado a cabo entre voluntarios pertenecientes a la Asociación Española de Creutzfeldt- 
Jakob, que agrupa a familiares y pacientes afectados por las enfermedades priónicas en España, reveló episodios de discriminación en la atención sanitaria motivados principalmente por el desconocimiento sobre estas patologías entre los profesionales que, al no conocer los protocolos de actuación, temen el trato con estos pacientes y los discriminan (Coca et al., 2019).

Otro de los aspectos sociosanitarios más importantes en las ER, y que también ilustran a la perfección las enfermedades priónicas, es la dificultad de llegar a un diagnóstico correcto en un plazo de tiempo razonable. Tal y como recoge FEDER, los pacientes de ER requieren de media 5 años para obtener un diagnóstico acertado y en un $20 \%$ de los casos los tiempos aumentan a 10 años o más. Esto hace que, en el caso de que haya algún tratamiento para la enfermedad en cuestión, este se administre tarde, generalmente tras un agravamiento de la situación del paciente. Además, un $26 \%$ de los casos reciben en algún momento un tratamiento inadecuado para su enfermedad (FEDER, n.d.). A su vez, las dificultades en el diagnóstico hacen que muchos de los pacientes que sufren de una ER hayan tenido que desplazarse en busca de diagnóstico y tratamiento adecuados, lo que incrementa los costes asociados a este tipo de enfermedades, además de tener un impacto negativo en el estado emocional de los pacientes. Todas estas circunstancias ocurren también en el caso de las enfermedades priónicas, cuyos síntomas son muy similares a los de otras enfermedades neurodegenerativas más comunes, lo que imposibilita obtener un diagnóstico definitivo en vida en la mayoría de los casos y hace por tanto necesaria una autopsia para la confirmación del mismo (Budka et al., 1995). La similitud de los síntomas, junto con el desconocimiento generalizado por parte de los profesionales sanitarios de estas enfermedades poco prevalentes, tiene por consiguiente un impacto significativo en la calidad asistencial que reciben los pacientes aquejados de ER, poniendo de relieve una vez más la importancia de la visibilización.

Aparte de los problemas con el diagnóstico, los gastos asociados a la atención de los afectados por ER han sido también largamente denunciados por pacientes y asociaciones. Uno de los mayores problemas a este respecto deriva de la escasa o nula cobertura de los productos sanitarios que requieren estos pacientes por la Sanidad Pública, así como del hecho de que en el $85 \%$ de los casos estos suponen tratamientos crónicos o de larga duración (FEDER, n.d.). Dada la baja frecuencia de este tipo de enfermedades, normalmente no son tenidas en cuenta al diseñar o modificar las políticas de salud pública, lo que repercute negativamente en el acceso de las familias afectadas a los medicamentos necesarios, en caso de que existan. De forma similar, la progresividad y carácter debilitante de muchas de las ER generan gastos que no se contemplan en el caso de otras patologías más prevalentes, como ayudas técnicas y ortopedia (requeridos en el 30\% de los casos de ER), transporte 
adaptado (27\% de los casos), asistencia personal (23\% de los casos) y adaptación de la vivienda ( $9 \%$ de los casos) (FEDER, n.d.). A pesar de que todos esos gastos son consecuencia directa de la enfermedad, la invisibilidad de las ER en el sistema público dificulta el acceso a ayudas destinadas a costear este tipo de gastos. En el caso de las EET, al tratarse de enfermedades neurodegenerativas de progresión rápida sin terapia disponible, la mayoría de los problemas asociados al gasto corresponden a ayudas técnicas, adaptación de transporte y vivienda y la contratación de asistencia personal. En este sentido, algunos países ofrecen ayudas a la dependencia asociadas a distintos grados de discapacidad, ya que, según FEDER, hasta un $70 \%$ de sus asociados tiene reconocido cierto grado de discapacidad. No obstante, en muchos casos el grado reconocido resulta insuficiente o, como en el caso de las enfermedades priónicas (cuya evolución es extraordinariamente rápida) (Johnson \& Gibbs, 1998), estas ayudas a la dependencia llegan con gran retraso y son insuficientes para hacer frente a una situación rápidamente cambiante. Así, el caso de las enfermedades priónicas evidencia que los mecanismos habituales para el apoyo y la protección de personas dependientes son insuficientes para las heterogéneas situaciones que plantean las distintas ER, y pone de manifiesto la necesidad de legislación específica que equipare la cobertura sociosanitaria de estos pacientes con la del resto de la población.

Afortunadamente, las últimas décadas han traído importantes avances en cuanto a la percepción de las enfermedades raras. El hecho de que, en su conjunto, las ER afecten aproximadamente a 300 millones de personas (Nguengang Wakap et al., 2020) ha hecho que algunos países o regiones centren su atención en ellas, convirtiendo el desarrollo de tratamientos en una prioridad de salud pública. Este fenómeno ha venido en gran parte impulsado por el movimiento asociativo surgido en torno a muchas de estas enfermedades, que ha sido más lento que en el caso de enfermedades más prevalentes debido a los pocos casos existentes y su gran dispersión geográfica. Es el caso principalmente de Europa y EEUU, donde ya en 1983 se aprobó el Orphan Drug Act (ODA) impulsado por la Organización Nacional para las Enfermedades Raras (NORD), uno de los hitos legislativos del siglo XXI, que recogía medidas para atender las necesidades médicas de los afectados por ER y estimular el desarrollo de terapias (Tambuyzer, 2010). El ODA reconocía que uno de los principales escollos para el desarrollo de estas terapias era el desinterés de la industria farmacéutica en realizar inversiones, que acarrearían en todos los casos muy bajos retornos debido al bajo número de pacientes. Por ello, esta legislación estableció una serie de incentivos económicos que incluían 7 años de exclusividad de mercado para los denominados fármacos huérfanos (Orphan Drugs) aprobados por la FDA (Food and Drug Administration de EEUU) destinados al tratamiento de enfermedades con baja prevalencia, exenciones de pago de las tasas de la FDA, 
asesoramiento gratuito para la fase de aprobación, y ventajas fiscales para los promotores e inversores (Tambuyzer, 2010). La Agencia del Medicamento europea (European Medicines Agency o EMA) se equiparó poco después con legislación propia en esta misma dirección y, más recientemente, algunos países asiáticos han puesto en marcha iniciativas legislativas similares con el objetivo de incentivar la investigación en ER (Song et al., 2012).

Finalmente, cabe destacar la importante labor de las asociaciones y agrupaciones de pacientes y familiares en los avances tanto a nivel social como sanitario. Muchas de las dificultades que comparten las ER, como la falta de información y conocimiento o la dispersión geográfica, pueden ser remediadas gracias a las asociaciones, que ofrecen información y apoyo a los afectados y realizan una labor inestimable de asesoramiento, orientación y consuelo, siendo el primer contacto para la gran mayoría de pacientes con otras personas en su misma situación. Además de mejorar significativamente la situación social y psicológica de los afectados por ER y sus familiares, las asociaciones realizan un trabajo de visibilización muy necesario, suministrando información tanto a los propios afectados como a la sociedad, y específicamente a los profesionales sanitarios. Por último, y sin olvidar la relevancia de las agrupaciones de pacientes y las asociaciones para impulsar cambios legislativos favorables a las ER, es necesario considerar el papel de los movimientos asociativos en la financiación de la investigación biomédica enfocada a las ER. Teniendo en cuenta que la solución a estas enfermedades pasa por impulsar su investigación, y que para ello se requiere una importante inversión que no es de interés para las grandes compañías farmacéuticas, las campañas de captación de fondos que llevan a cabo las asociaciones resultan de vital importancia para que se produzcan avances en este terreno.

En conclusión, las enfermedades priónicas humanas, junto con el resto de las enfermedades raras, requieren primero una mayor visibilidad y segundo, la acción coordinada de administraciones, asociaciones de pacientes, investigadores y en definitiva del conjunto de la sociedad, para seguir avanzando en la dirección emprendida en los años 80 y dar respuesta a las necesidades y desafíos particulares que suponen todas y cada una de las enfermedades de baja prevalencia, mal llamadas enfermedades raras. 


\section{Referencias bibliográficas:}

Alper, T. (1972). The nature of the scrapie agent. Journal of Clinical Pathology. Supplement (Royal College of Pathologists), 6, 154-155.

Benestad, S. L., et al. (2016). First case of chronic wasting disease in europe in a norwegian free-ranging reindeer. Veterinary Research, 47(1), 88-0160375-4. doi:10.1186/s13567-016-0375-4

Bonda, D. J., et al. (2016). Human prion diseases: Surgical lessons learned from iatrogenic prion transmission. Neurosurgical Focus, 41(1), E10. doi:10.3171/2016.5.FOCUS15126

Brown, P. (2009). Reflections on a half-century in the field of transmissible spongiform encephalopathy. Folia Neuropathologica, 47(2), 95-103. doi: 12704

Brown, P., et al. (2012). Iatrogenic creutzfeldt-jakob disease, final assessment. Emerging Infectious Diseases, 18(6), 901-907. doi:10.3201/ eid1806.120116

Budka, H., et al. (1995). Neuropathological diagnostic criteria for creutzfeldtjakob disease (CJD) and other human spongiform encephalopathies (prion diseases). Brain Pathology, 5(4), 459-466.

Bueler, H., et al. (1993). Mice devoid of PrP are resistant to scrapie. Cell, 73(7), 1339-1347. doi:0092-8674(93)90360-3

Coca, J. R., et al. (2019). Análisis cualitativo del impacto social y familiar de las encefalopatías espongiformes transmisibles humanas. Revista De Neurología, 69, 242-248. doi:10.33588/ rn.6906.2019122

Cohen, F. E., \& Prusiner, S. B. (1998). Pathologic conformations of prion proteins. Annual Review of Biochemistry, 67, 793-819. doi:10.1146/ annurev.biochem.67.1.793

Collinge, J., et al. (1996). Molecular analysis of prion strain variation and the aetiology of 'new variant' CJD. Nature, 383(6602), 685-690. doi: $10.1038 / 383685 \mathrm{a} 0$

Creutzfeldt, H. (1920). Über eine eigenartige herdförmige erkrankung des zentralnervensystems. Z Gesamte Neurol Psychiatr, 57, 1-19.

Chandler, R. L. (1961). Encephalopathy in mice produced by inoculation with scrapie brain material. Lancet, 1(7191), 1378-1379. doi:10.1016/s01406736(61)92008-6

Chesebro, B., et al. (1985). Identification of scrapie prion protein-specific mRNA in scrapie-infected and uninfected brain. Nature, 315(6017), 331333. doi:10.1038/315331a0

Duffy, P., et al. (1974). Letter: Possible person-to-person transmission of creutzfeldt-jakob disease. The New England Journal of Medicine, 290(12), 692-693. 
Duque Velasquez, C., et al. (2015). Deer prion proteins modulate the emergence and adaptation of chronic wasting disease strains. Journal of Virology, 89(24), 12362-12373. doi:10.1128/JVI.02010-15

Eraña, H., et al. (2017). Prion-like disorders and transmissible spongiform encephalopathies: An overview of the mechanistic features that are shared by the various disease-related misfolded proteins. Biochemical and Biophysical Research Communications, 483(4), 1125-1136. doi:S0006291X(16)31430-9

European Commission. (n.d.). Rare diseases. retrieved 2020, nov 7. Https:// Ec.Europa.Eu/Health/Non_communicable_diseases/Rare_diseases_en,

FEDER. (n.d.). Federación española de enfermedades raras. Https:// Enfermedades-Raras.Org/,

Gajdusek, D. C. (1967). Slow-virus infections of the nervous system. The New England Journal of Medicine, 276(7), 392-400. doi:10.1056/ NEJM196702162760708

Gajdusek, D. C., et al. (1967). Transmission and passage of experimenal "kuru" to chimpanzees. Science (New York, N.Y.), 155(3759), 212-214.

Gajdusek, D. C., et al. (1966). Experimental transmission of a kuru-like syndrome to chimpanzees. Nature, 209(5025), 794-796.

Gambetti, P., et al. (2008). A novel human disease with abnormal prion protein sensitive to protease. Annals of Neurology, 63(6), 697-708. doi:10.1002/ ana. 21420

Gambetti, P., et al. (2003). Sporadic and familial CJD: Classification and characterisation. British Medical Bulletin, 66, 213-239. doi:10.1093/ $\mathrm{bmb} / 66.1 .213$

Gibbs, C. J.,Jr, et al. (1968). Creutzfeldt-jakob disease (spongiform encephalopathy): Transmission to the chimpanzee. Science, 161(3839), 388-389. doi:10.1126/science.161.3839.388

Glatzel, M., et al. (2005). Human prion diseases: Molecular and clinical aspects. Archives of Neurology, 62(4), 545-552. doi:62/4/545

Hadlow, W. J. (2008). Kuru likened to scrapie: The story remembered. Philosophical Transactions of the Royal Society of London.Series B, Biological Scis, 363(1510), 3644. doi:10.1098/rstb.2008.4013

Hill, A. F., et al. (1997). The same prion strain causes vCJD and BSE. Nature, 389(6650), 448-50, 526. doi:10.1038/38925

Hill, A. F., et al. (2003). Molecular classification of sporadic creutzfeldt-jakob disease. Brain, 126(Pt 6), 1333-1346.

Houston, F., \& Andreoletti, O. (2019). Animal prion diseases: The risks to human health. Brain Pathology (Zurich, Switzerland), 29(2), 248-262. doi:10.1111/bpa.12696 
Huor, A., et al. (2019). The emergence of classical BSE from atypical/Nor98 scrapie. Proceedings of the National Academy of Sciences of the United States of America, doi:201915737

Jakob, A. (1921). Über eigenartige erkrankungen des zentralnervensystems mit bemerkenswertem anatomischem. befunde. (spastische pseudoskleroseencephalomyelopathie mit disseminierten degenerationsherden). $Z$ Gesamte Neurol Psychiatr, 64, 147-228.

Johnson, R. T., \& Gibbs, C. J.,Jr. (1998). Creutzfeldt-jakob disease and related transmissible spongiform encephalopathies. The New England Journal of Medicine, 339(27), 1994-2004. doi:10.1056/NEJM199812313392707

Klatzo, I., et al. (1959). Pathology of kuru. Laboratory Investigation; a Journal of Technical Methods and Pathology, 8(4), 799-847.

Konold, T., et al. (2004). Clinical findings in 78 suspected cases of bovine spongiform encephalopathy in great britain. The Veterinary Record, 155(21), 659-666. doi:10.1136/vr.155.21.659

Kovacs, G. G., et al. (2005). Genetic prion disease: The EUROCJD experience. Human Genetics, 118(2), 166-174. doi:10.1007/s00439-005-0020-1

Kretzschmar, H. A., et al. (1995). Codon 178 mutation of the human prion protein gene in a german family (backer family): Sequencing data from 72-year-old celloidin-embedded brain tissue. Acta Neuropathologica, 89(1), 96-98.

Latarjet, R., et al. (1970). Inactivation of the scrapie agent by near monochromatic ultraviolet light. Nature, 227(5265), 1341-1343. doi:10.1038/2271341a0

Liberski, P. P. (2012). Historical overview of prion diseases: A view from afar. Folia Neuropathologica, 50(1), 1-12. doi:18385

Liberski, P. P., et al. (2019). Kuru, the first human prion disease. Viruses, 11(3), 10.3390/v11030232. doi:E232

Linsell, L., et al. (2004). A case-control study of sporadic creutzfeldt-jakob disease in the united kingdom: Analysis of clustering. Neurology, 63(11), 2077-2083. doi:63/11/2077

Mathiason, C.K. (2017). Scrapie, CWD, and transmissible mink encephalopathy. Progress in Molecular Biology and Translational Science, 150, 267-292. doi:S1877-1173(17)30119-9

Minikel, E. V., et al. (2016). Quantifying prion disease penetrance using large population control cohorts. Science Translational Medicine, 8(322), doi:10.1126/scitranslmed.aad5169

Nguengang Wakap, S., et al. (2020). Estimating cumulative point prevalence of rare diseases: Analysis of the orphanet database. European Journal of Human Genetics : EJHG, 28(2), 165-173. doi:10.1038/s41431-0190508-0 
Notari, S., et al. (2014). Transmission characteristics of variably proteasesensitive prionopathy. Emerging Infectious Diseases, 20(12), 2006-2014. doi:10.3201/eid2012.140548

Pattison, I. H., \& Millson, G. C. (1961). Scrapie produced experimentally in goats with special reference to the clinical syndrome. Journal of Comparative Pathology, 71, 101-109.

Prusiner, S. B. (1982). Novel proteinaceous infectious particles cause scrapie. Science, 216(4542), 136-144.

Prusiner, S. B. (1989). Scrapie prions. Annual Review of Microbiology, 43, 345374. doi:10.1146/annurev.mi.43.100189.002021

Saborio, G. P., et al. (2001). Sensitive detection of pathological prion protein by cyclic amplification of protein misfolding. Nature, 411(6839), 810-813. doi: $10.1038 / 35081095$

Song, P., et al. (2012). Rare diseases, orphan drugs, and their regulation in asia: Current status and future perspectives. Intractable \& Rare Diseases Research, 1(1), 3-9. doi:10.5582/irdr.2012.v1.1.3

Takada, L. T., et al. (2017). Genetic prion disease: Experience of a rapidly progressive dementia center in the united states and a review of the literature. American Journal of Medical Genetics. Part B, Neuropsychiatric Genetics, 174(1), 36-69. doi:10.1002/ajmg.b.32505

Tambuyzer, E. (2010). Rare diseases, orphan drugs and their regulation: Questions and misconceptions. Nature Reviews.Drug Discovery, 9(12), 921-929. doi:10.1038/nrd3275

Tesar, A., et al. (2019). Clinical variability in P102L gerstmann-strausslerscheinker syndrome. Annals of Neurology, 86(5), 643-652. doi:10.1002/ ana.25579

Thadani, V., et al. (1988). Creutzfeldt-jakob disease probably acquired from a cadaveric dura mater graft. case report. Journal of Neurosurgery, 69(5), 766-769. doi:10.3171/jns.1988.69.5.0766

Verbeke, W. (2001). Consumer reactions and economic consequences of the BSE crisis. Verhandelingen - Koninklijke Academie Voor Geneeskunde Van Belgie, 63(5), 483-492.

Wang, F., et al. (2010). Generating a prion with bacterially expressed recombinant prion protein. Science, 327(5969), 1132-1135. doi:10.1126/ science. 1183748

Wickner, R. B., et al. (2015). Yeast prions: Structure, biology, and prionhandling systems. Microbiology and Molecular Biology Reviews : MMBR, 79(1), 1-17. doi:10.1128/MMBR.00041-14

Williams, E. S. (2005). Chronic wasting disease. Veterinary Pathology, 42(5), 530-549. doi:42/5/530 
Yuan, A. H., \& Hochschild, A. (2017). A bacterial global regulator forms a prion. Science (New York, N.Y.), 355(6321), 198-201. doi:10.1126/science. aai7776

Zlotnik, I., \& Rennie, J. C. (1965). Experimental transmission of mouse passaged scrapie to goats, sheep, rats and hamsters. Journal of Comparative Pathology, 75, 147-157. 
